\title{
State Space System Modeling of a Quad Copter UAV
}

\author{
Zaid Tahir*, Mohsin Jamil, Saad Ali Liaqat, Lubva Mubarak, Waleed Tahir and Syed Omer Gilani \\ School of Mechanical and Manufacturing Engineering (SMME), National University of Sciences and Technology \\ (NUST), H-12 Main Campus, Islamabad, Pakistan; \\ zaid.butt.tahir@gmail.com,mohsin@smme.nust.edu.pk,sa.liaqat@gmail.com, lub.mubarak@gmail.com, \\ waleedsmailbox@gmail.com, omer@smme.nust.edu.pk
}

\begin{abstract}
Background/Objectives: To derive a linear mathematical modeling of a quad-copter Unmanned Aerial Vehicle (UAV). Methods/Statistical Analysis: The quad-copter physical system with different inputs and outputs has been represented in a state-space form. State space representation is a mathematical model of a physical system as a set of input, output and state variables related by first-order differential equations. "State space" is the space which has state variables as its axes. Vectors are used within state space to show the state of the system. Findings: The six Degrees Of Freedom (6DOF) quad copter state-space model is developed starting from basic Newtonian equations, which is very important to control the quad copter system. The system of quadcopter aerial vehicle is inherently dynamically unstable, which means that this study has found out the state-space modeling. Therefore, this study has enabled the successful modelling of the quadcopter system, which directly contributes to the stable flight of the unmanned aerial vehicle. Application/Improvements: Unmanned aerial reconnaissance, autonomous aerial flight.
\end{abstract}

Keywords: Quad-copter Unmanned Aerial Vehicle, State Space Modeling, Unmanned Aerial Vehicle

\section{Introduction}

In the most recent couple of decades fast improvement has been completed in multi-mission proficient, small unmanned aerial vehicles. They are picking up significance because of their capacity to replace manned aerial vehicles in standard and additionally hazardous missions, which thus diminish expenses of numerous aerial operations. These aerial vehicles are being utilized in different nonmilitary missions, for example, news agencies, climate checking and by law authorization offices. Thus they are likewise being widely utilized as a part of military operations, for example, insight information gathering, observation and surveillance missions, and aerial targeting. These flexible applications have prompted a propelled research for expanding the level of self-sufficiency of these unmanned aerial vehicles. On the premise of their configuration unmanned aerial vehicles are separated into two primary classes: fixed-wing and rotary-wing UAVs

Fixed wing UAVs is the most widely recognized sort. Their configuration is straightforward when contrasted with different sorts and they can fly for long lengths of time at high speeds. Nonetheless, these UAVs need runways or other recovery and launch frameworks like sling or parachute for takeoff and landing. Fixed wing UAVs are not suitable to be flown indoors, at low altitudes. Rotary wing UAVs, are better because of their absence of requirement for runways for takeoff and landing which makes them perfect for use in tight and congested regions. Because of their high mobility, small size and hovering capacities, rotary wing UAVs are favored.

\section{Working Principle}

A Quad Copter is a four-rotor helicopter. It is an underactuated, dynamic vehicle with four input forces (one for each rotor) and six Degrees Of Freedom (6DOF). The motion of a Quad copter in 6DOF is controlled by varying the rpm of the four rotors individually, thereby changing the lift and rotational forces. Quad copter tilts toward the direction of slow spinning motor, which enables it to roll

*Author for correspondence 
and pitch. Roll and pitch angles divides the thrust into two directions due to which linear motion is achieved. The rotors rotate in clockwise- anticlockwise pairs, shown in Figure 1 to control the yaw produced due to the drag force on propellers. The Center of Gravity (CG) lies almost at the same plane which contains all the rotors. Also all four motors of same class differ in efficiency with each other. This differentiates it from helicopters and it is very difficult to stabilize a quad copter by human control. Therefore a sophisticated control is essential for a balance flight of quad copter.

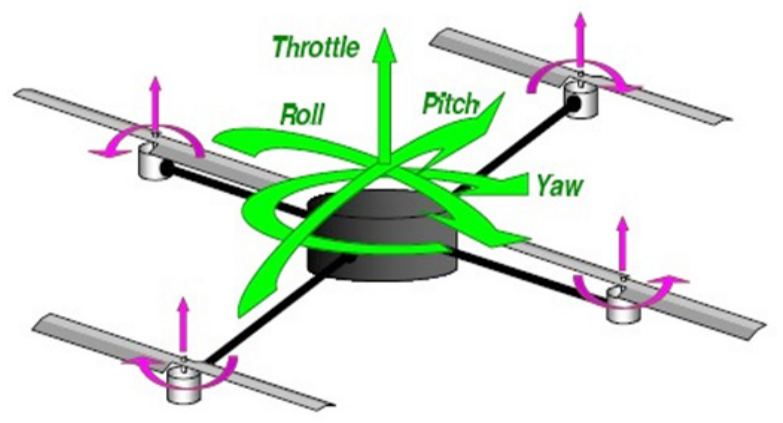

Figure 1. Quad copter schematic.

\section{Quad Copter Modeling}

\subsection{Quad Copter Dynamics}

The motion of Quad Copter in 6DOF is controlled by varying the rpm of four rotors individually, thereby changing the vertical, horizontal and rotational forces as depicted in Figure 2.

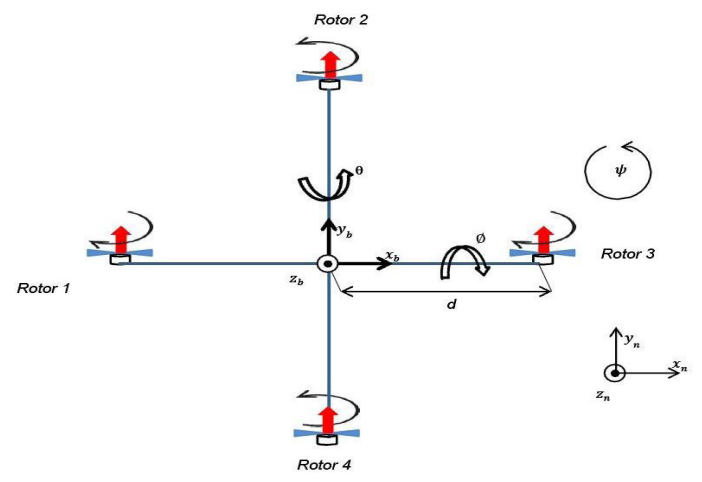

Figure 2. Quad copter dynamics.

\subsubsection{Roll Motion}

The x-axis torque $\tau_{x}$ i.e. rolling moment. Where " $\mathrm{r}$ " is the moment arm of the individual forces.

$$
r_{x}=\sum_{i=1}^{4} r_{i} f_{i}
$$

$$
\tau_{x}=r_{1} f_{1}+r_{2} f_{2}+r_{3} f_{3}+r_{4} f_{4}
$$

The first and the third rotors are on the $\mathrm{x}$-axis, hence their moment arm is zero and the rolling moment contributed by them is also zero as shown in Figure 2. The second rotor will produce a positive moment (counter clockwise). The fourth rotor will produce a negative moment (clockwise).

$r_{\mathbf{2}}=d$ and $r_{\mathbf{4}}=-d$

So Equation 2 becomes:

$\tau_{x}=d f_{2}-d f_{4}$

\subsubsection{Pitch Motion}

The y-axis torque $\tau_{y}$ i.e. pitch. and " $r$ " is the moment arm of the forces from y-axis. From Equation 1, we get:

$$
\begin{aligned}
& \tau_{y}=r_{1} f_{1}+r_{2} f_{2}+r_{3} f_{3}+\mathbf{r}_{4} \mathbf{f}_{4} \\
& r_{2}=r_{4}=\mathbf{0} \text { and } r_{1}=-r_{3}=d
\end{aligned}
$$

So Equation 3 becomes:

$\tau_{\mathbf{y}}=\mathrm{df}_{1}-\mathbf{d f}_{3}$

\subsubsection{Yaw Motion}

The z-axis torque $\tau_{z}$ i.e. yaw (Figure 2 and 3). Reactive torque due to air drag on the rotor blade is encountered by the motor as they rotate. This reactive torque is modeled by $\mathrm{Q}$. The imbalance between the rotating propellers creates the yaw. Along the counterclockwise rotation this

$$
\tau_{z}=\sum_{i=1}^{4} Q_{i}
$$

Whereas $Q=c F_{i}$ if rotation of the $i^{i^{\text {th }}}$ rotor is counterclockwise and $-c F_{i}$, if clockwise. So opening the summation becomes:

$$
\tau_{z}=c\left(-F_{1}+F_{2}-F_{3}+F_{4}\right)
$$

Where ' $c$ ' in Equation 7 is the force to moment scaling factor for a fixed motor-propeller set and can be calculated experimentally ${ }^{1}$.

\subsubsection{Vertical Motion}

The vertical motion of the quad copter is governed by the total thrust force ' $T$ ' and weight ' $w$ ' along the $\mathrm{z}$-axis shown in Figure 2. The rpms of the four rotating propellers are changed simultaneously in order to accomplish vertical motion. 


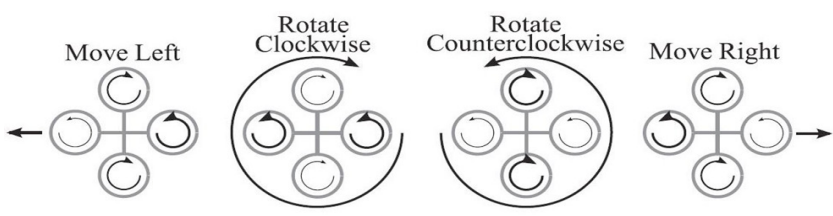

Figure 3. Yaw and linear motion due to rotor forces.

$$
T=\sum_{i=1}^{4} F_{i}
$$

The vertical motion is described by the equation (Newton's $2^{\text {nd }}$ law):

$$
z^{\prime \prime}=\frac{T-m g}{m}
$$

\subsubsection{Horizontal Motion}

The horizontal motion in the $\mathrm{x}$ and $\mathrm{y}$-axes is accomplished by decreasing the rpm of the rotor in whose direction it is intended to move, and subsequently increasing the rpm of the rotor on the opposite side of the same arm as depicted in Figure 3. The force imbalance will cause the quad copter to tilt to one side and the horizontal component of the thrust force will impart horizontal linear motion to the quad copter shown Figure 4. If the mass of the quad copter is ' $m$ ', then referring to Figure 4, the linear acceleration in the horizontal $\mathrm{x}$-direction can be written as:

$$
\boldsymbol{x}^{\prime^{\prime}}=-\frac{T \cos \theta}{m}
$$

Using small angle approximation $(\theta<0.5 \mathrm{rad})$ :

$$
\sin \theta \approx \theta
$$

Since $\theta$ is small:

$$
T \approx m g
$$

Thus, from equation 10 and 11, equation 9 becomes:

$$
\boldsymbol{x}^{\prime^{\prime}}=-\boldsymbol{g} \boldsymbol{\theta}
$$

Similarly, $\bar{y}$ can be derived out to be:

$$
y^{\prime^{\prime}}=g \phi
$$

Thus Equation 3, 5, 7, 9, 13 and 14 give us the dynamics of the quad copter in all 6 degrees of freedom. After the dynamical equations have been established, we can move towards the state space modeling of the quad rotor.

\subsection{State Space Representation}

A state space representation is a mathematical model of a physical system as a set of input, output and state vari- ables related by first-order differential equations. "State space" is the space which has state variables as its axes. Vectors are used within state space to show the state of the system. Generally, state-space representation of a linear system with $\mathrm{p}$ inputs, $\mathrm{q}$ outputs and $\mathrm{n}$ state variables is written in the following form

$$
\begin{aligned}
& \dot{x}(\mathrm{t})=\mathrm{Ax}(\mathrm{t})+\Xi \mathrm{u}(\mathrm{t}) \\
& \mathrm{y}(\mathrm{t})=\mathrm{Cx}(\mathrm{t})+\mathrm{Du}(\mathrm{t})
\end{aligned}
$$

Where,

$$
\begin{aligned}
& \boldsymbol{x}(\boldsymbol{t})=\text { 'State Vector' } \quad \boldsymbol{y}(\boldsymbol{t})=\text { 'Output Vector' } \\
& \boldsymbol{u}(\boldsymbol{t})=\text { 'Input (or control) Vector' } \\
& \mathrm{A}=\text { 'System Matrix' } \\
& \mathrm{B}=\text { 'Input Matrix' } \\
& \mathrm{C}=\text { 'Output Matrix' } \\
& \mathrm{D}=\text { ='Feed forward Matrix' } \\
& \dot{x}(\mathrm{t})=\frac{d}{d t} x(t)
\end{aligned}
$$

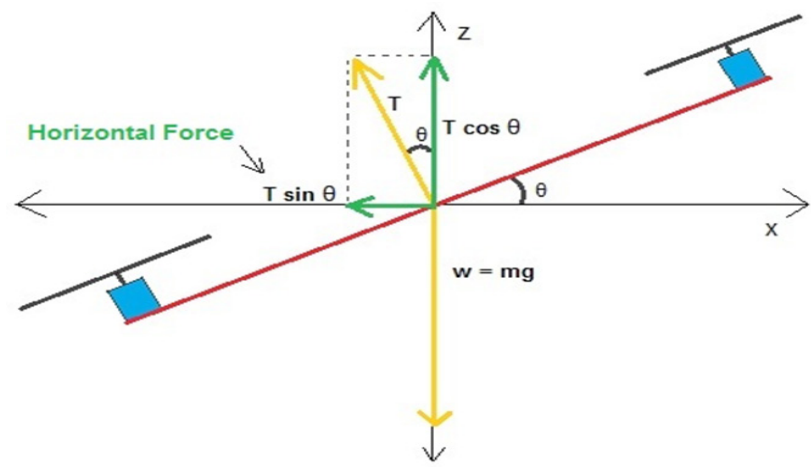

Figure 4. Horizontal motion.

\subsection{DOF Quad Copter Modeling}

We start by selecting the system states. As seen in literature ${ }^{2-7}$, the following twelve states are practically suitable for a quad copter in $6 \mathrm{DOF}$.

- Position along $\mathrm{x}$ axis - $x$

- Position along y axis - $y$

- Position along $\mathrm{z}$ axis (height) - $z$

- Velocity along x axis - $x^{\prime}$

- Velocity along y axis - $y^{\prime}$

- Velocity along $\mathrm{z}$ axis - $z^{\prime}$

- Roll angle - $\phi$

- Pitch angle - $\theta$

- Yaw angle - $\psi$

- Roll rate - $\phi^{\prime}$

- Pitch rate $-\theta^{\prime}$

- Yaw rate - $\psi^{\prime}$ 
Thus, we will get our state vector $\boldsymbol{x}$ as follows:

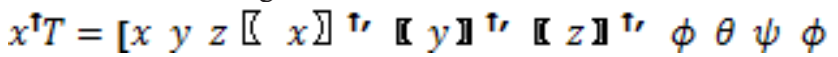

\section{, 【 $\theta \rrbracket^{\top}{ }^{\prime}$ 【 $\left.\psi \rrbracket^{\top}\right]$}

The input matrix $\boldsymbol{u}$ is given in Equation 21 as follows:

$u^{\uparrow} T=\left[\begin{array}{llll}U_{1} 1 & U_{2} & U_{3} & U_{4}\end{array}\right]$

Where,

- $U_{1}$ is the Total Upward Force on the quad rotor along $\mathrm{z}$-axis $(T-m g)$

- $U_{\mathbf{2}}$ is the Pitch Torque (about $\mathrm{x}$-axis)

- $U_{3}$ is the Roll Torque (about y-axis)

- $U_{\mathbf{4}}$ is the Yaw Torque (about $\mathrm{z}$-axis)

The output matrix $\boldsymbol{y}$ is given as follows:

$y^{\mathbf{\uparrow}} T=\left[\begin{array}{llllll}x & y & z & \phi & \theta & \psi\end{array}\right]$

From Equations 3, 5, 7, 9, 13, and 14 the state differential equations can be written as:

$$
\begin{aligned}
& x^{\prime}=x^{\prime} \\
& y^{\prime}=y^{\prime} \\
& z^{\prime}=z^{\prime} \\
& x{ }^{\prime \prime}=-g \theta \\
& y^{\prime^{\prime}}=-\frac{U_{1}}{m} \\
& z^{\prime^{\prime}}=-\phi^{\prime} \\
& \phi^{\prime}=\phi^{\prime} \\
& \theta^{\prime}=\theta^{\prime} \\
& \psi^{\prime}=\dot{U}_{\mathbf{2}} \\
& \phi \llbracket \overline{U_{3} x} \\
& \theta^{\prime^{\prime}}=\frac{U_{4}}{I_{z}} \\
& \psi^{\prime^{\prime}}=\frac{{ }^{\prime}}{}
\end{aligned}
$$

The above state differential equations written in matrix form are shown as Equation 20 as follows. A one to one correspondence has been done with Equation 15 for the extraction of state space matrices.

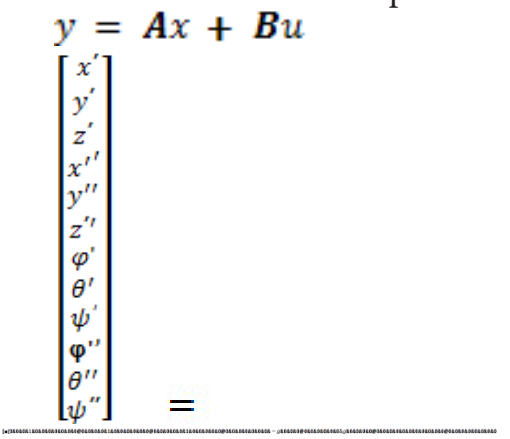

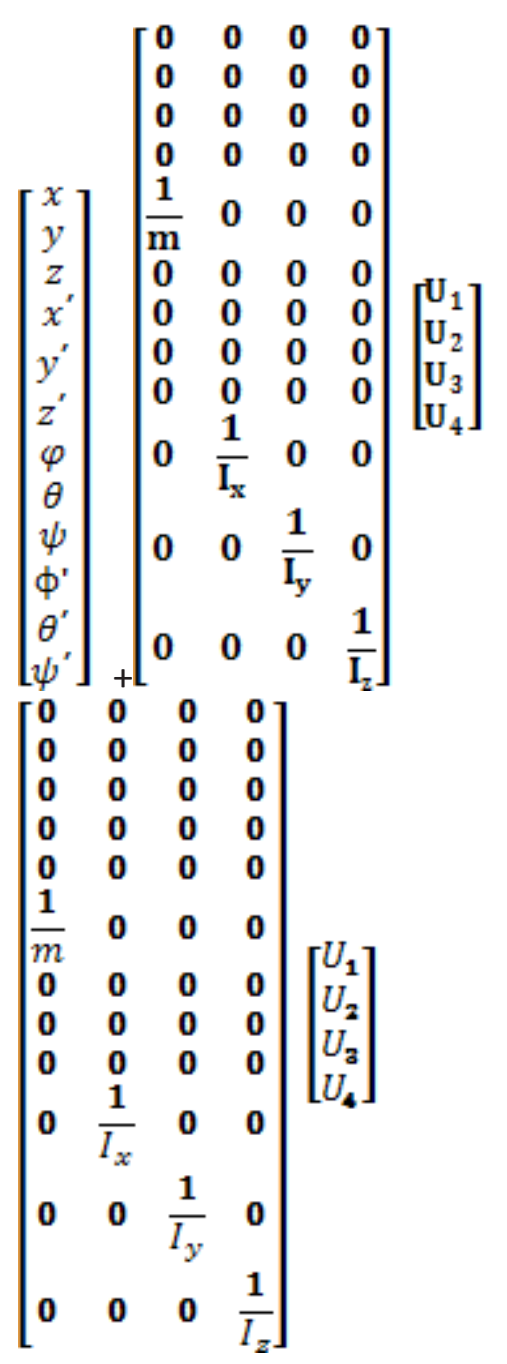

Comparing equation 20 with Equation 15, we can observe that:

$A=$

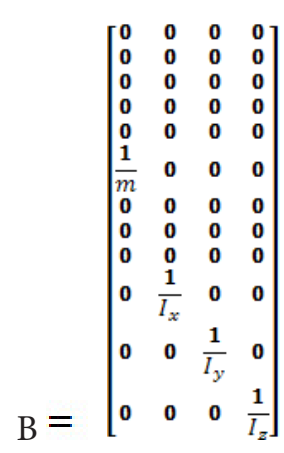

Comparing the output equation 21 with equation 16 , we have:

$$
y=\boldsymbol{C} x+\boldsymbol{D} u
$$




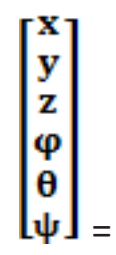

$\left[\begin{array}{llllllllllll}\mathbf{1} & \mathbf{0} & \mathbf{0} & \mathbf{0} & \mathbf{0} & \mathbf{0} & \mathbf{0} & \mathbf{0} & \mathbf{0} & \mathbf{0} & \mathbf{0} & \mathbf{0} \\ \mathbf{0} & \mathbf{1} & \mathbf{0} & \mathbf{0} & \mathbf{0} & \mathbf{0} & \mathbf{0} & \mathbf{0} & \mathbf{0} & \mathbf{0} & \mathbf{0} & \mathbf{0} \\ \mathbf{0} & \mathbf{0} & \mathbf{1} & \mathbf{0} & \mathbf{0} & \mathbf{0} & \mathbf{0} & \mathbf{0} & \mathbf{0} & \mathbf{0} & \mathbf{0} & \mathbf{0} \\ \mathbf{0} & \mathbf{0} & \mathbf{0} & \mathbf{0} & \mathbf{0} & \mathbf{0} & \mathbf{1} & \mathbf{0} & \mathbf{0} & \mathbf{0} & \mathbf{0} & \mathbf{0} \\ \mathbf{0} & \mathbf{0} & \mathbf{0} & \mathbf{0} & \mathbf{0} & \mathbf{0} & \mathbf{0} & \mathbf{1} & \mathbf{0} & \mathbf{0} & \mathbf{0} & \mathbf{0} \\ \mathbf{0} & \mathbf{0} & \mathbf{0} & \mathbf{0} & \mathbf{0} & \mathbf{0} & \mathbf{0} & \mathbf{0} & \mathbf{1} & \mathbf{0} & \mathbf{0} & \mathbf{0}\end{array}\right]$

$\left[\begin{array}{llll}\mathbf{0} & \mathbf{0} & \mathbf{0} & \mathbf{0} \\ \mathbf{0} & \mathbf{0} & \mathbf{0} & \mathbf{0} \\ \mathbf{0} & \mathbf{0} & \mathbf{0} & \mathbf{0} \\ \mathbf{0} & \mathbf{0} & \mathbf{0} & \mathbf{0} \\ \mathbf{0} & \mathbf{0} & \mathbf{0} & \mathbf{0} \\ \mathbf{0} & \mathbf{0} & \mathbf{0} & \mathbf{0}\end{array}\right]\left[\begin{array}{l}\mathrm{U}_{1} \\ \mathrm{U}_{2} \\ \mathrm{U}_{3} \\ \mathrm{U}_{4}\end{array}\right]$

$\left[\begin{array}{c}\mathrm{x} \\ \mathrm{y} \\ \mathrm{z} \\ \mathrm{x}^{\prime} \\ \mathrm{y}^{\prime} \\ \mathrm{z}^{\prime} \\ \varphi \\ \boldsymbol{\theta} \\ \psi \\ \varphi^{\prime} \\ \boldsymbol{\theta}^{\prime} \\ \psi^{\prime}\end{array}\right]_{+}$

Thus,

$\left[\begin{array}{llllllllllll}\mathbf{1} & \mathbf{0} & \mathbf{0} & \mathbf{0} & \mathbf{0} & \mathbf{0} & \mathbf{0} & \mathbf{0} & \mathbf{0} & \mathbf{0} & \mathbf{0} & \mathbf{0} \\ \mathbf{0} & \mathbf{1} & \mathbf{0} & \mathbf{0} & \mathbf{0} & \mathbf{0} & \mathbf{0} & \mathbf{0} & \mathbf{0} & \mathbf{0} & \mathbf{0} & \mathbf{0} \\ \mathbf{0} & \mathbf{0} & \mathbf{1} & \mathbf{0} & \mathbf{0} & \mathbf{0} & \mathbf{0} & \mathbf{0} & \mathbf{0} & \mathbf{0} & \mathbf{0} & \mathbf{0} \\ \mathbf{0} & \mathbf{0} & \mathbf{0} & \mathbf{0} & \mathbf{0} & \mathbf{0} & \mathbf{1} & \mathbf{0} & \mathbf{0} & \mathbf{0} & \mathbf{0} & \mathbf{0} \\ \mathbf{0} & \mathbf{0} & \mathbf{0} & \mathbf{0} & \mathbf{0} & \mathbf{0} & \mathbf{0} & \mathbf{1} & \mathbf{0} & \mathbf{0} & \mathbf{0} & \mathbf{0} \\ \mathbf{0} & \mathbf{0} & \mathbf{0} & \mathbf{0} & \mathbf{0} & \mathbf{0} & \mathbf{0} & \mathbf{0} & \mathbf{1} & \mathbf{0} & \mathbf{0} & \mathbf{0}\end{array}\right]$

$D=\left[\begin{array}{llll}0 & 0 & 0 & 0 \\ 0 & 0 & 0 & 0 \\ 0 & 0 & 0 & 0 \\ 0 & 0 & 0 & 0 \\ 0 & 0 & 0 & 0 \\ 0 & 0 & 0 & 0\end{array}\right]$
From Equations 20 and 21, we have the $\boldsymbol{A}, \boldsymbol{B}, \boldsymbol{C}$ and $\boldsymbol{D}$ matrices which completely define the 6DOF state space model of a quad copter.

\section{Conclusion}

6DOF state space model of the quad copter have been derived from basic Newtonian equations in this paper. The quad copter mathematical model derived in this paper is unique in the sense that it gives the reader the grass root level understanding of the dynamics of the quad copter UAV in 6DOF dynamical system. The models derived here will be later used for designing control laws for the $6 \mathrm{DOF}$ stability of the quad copter.

\section{References}

1. Bora E, Erdinc A. Modeling and PD control of a Quadrotor VTOL vehicle. IEEE Intelligent Vehicles Symposium; Istanbul, Turkey. 2007 Jun 13-15.

2. Balas C. Modelling and linear control of a quadrotor $[\mathrm{MSc}$ Thesis]. Cranfield University; 2007.

3. Mian AA, Daobo W. Modeling and back stepping-based nonlinear control strategy for a 6DOF quadrotor helicopter.

4. Totu MC, Koldbaek SK. Modelling and control of autonomous quad-rotor [Masters Thesis]. 2010.

5. Bresciani T. Modelling, identification and control of a quadrotor helicopter [Masters Thesis]. 2008 Oct.

6. de Oliveira MDC. Modeling, identification and control of a quadrotor aircraft [Masters Thesis]. 2011 Jun.

7. Salih AL, Moghavvemi M, Mohamed HAF, Gaeid KS. Modelling and PID controller design for a quadrotor unmanned air vehicle. 